\title{
RANKL subcellular trafficking in osteocytes
}

\section{OMasashi Honma, Yuki Ikebuchi, Yoshiaki Kariya, Madoka Hayashi, Naoki Hayashi, Shigeki Aoki and Hiroshi Suzuki \\ (Department of Pharmacy, The University of Tokyo Hospital)}

\section{Background}

Bone remodeling maintains bone quantity and quality, and receptor activator of the NF-KB ligand (RANKL) is the central player in the regulation of osteoclastogenesis.

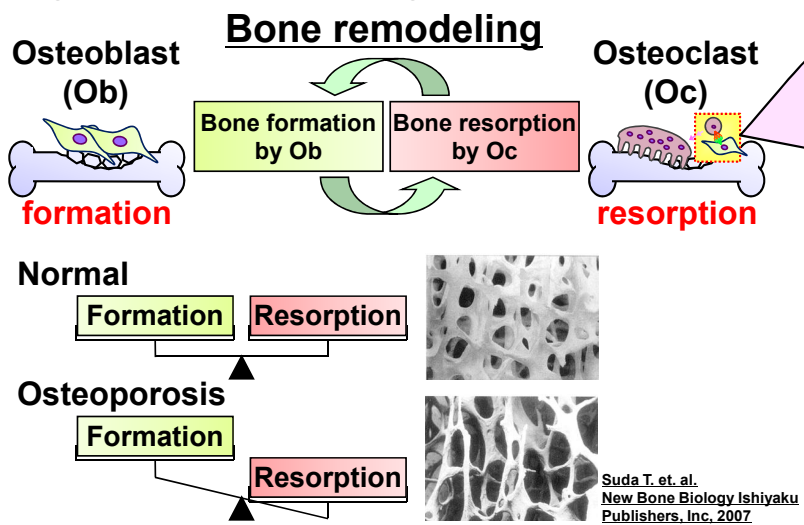

Conventional concept ( 2011)

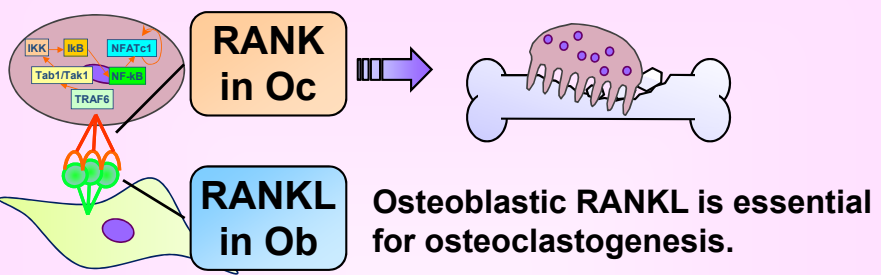

Novel hypothesis

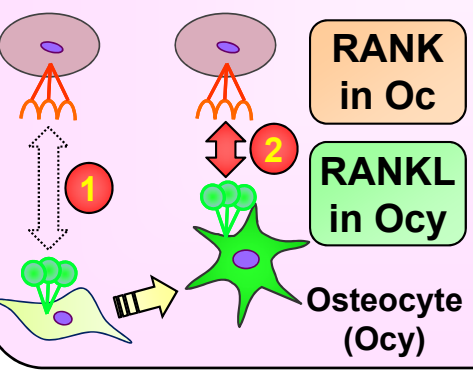

Sema4d from osteoclasts pushes absence of cell-cell interactions. Deletion of RANKL in osteocytes led to the drastic suppression of Nat Med. 2011.17(10):1231-34. $\square$

Osteocytes act as the major source of RANKL.)

\section{Purpose}

Elucidating how osteocytic RANKL is presented to RANK expressed in osteoclast

Soluble form? or

Direct interaction?

\section{Scheme}

1. Establishing a co-culture system of osteocytes and BMMs to mimic the physiological situation

2. Revealing RANKL signal delivery mechanism - soluble form ? or direct interaction? -

3. Examining the roles of regulatory machineries of RANKL traffic

\section{3D culture system of primary Osteocyte}

\section{Preparation of Ocy-rich population}

Ocys were isolated from calvarias of C57BL6 mice and the purity was 1. 2 confirmed by immuno-staining.

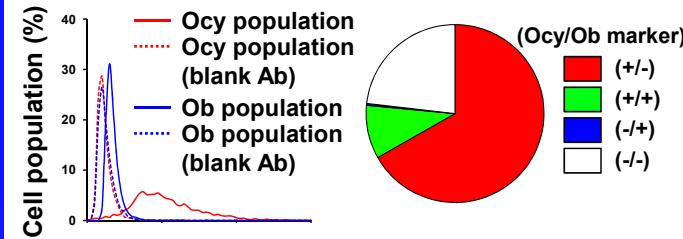

Culture condition of primary Ocys

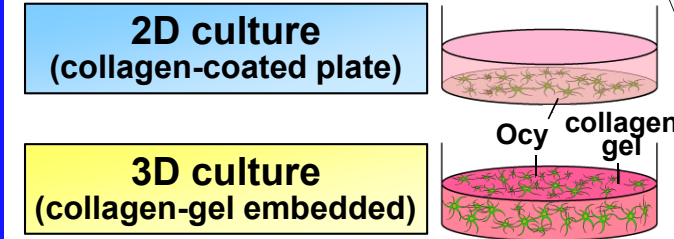

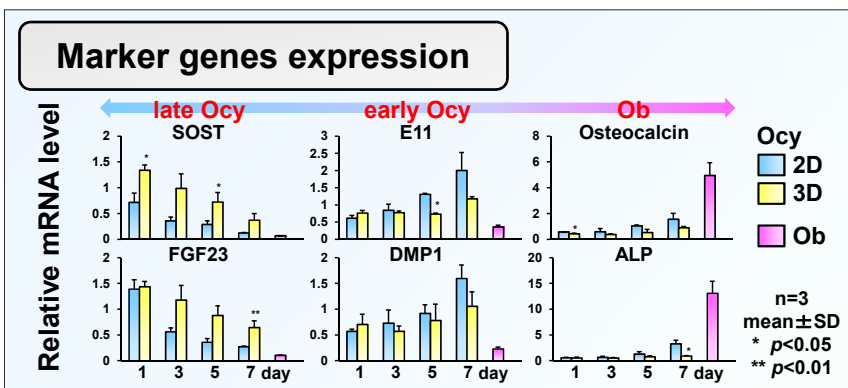

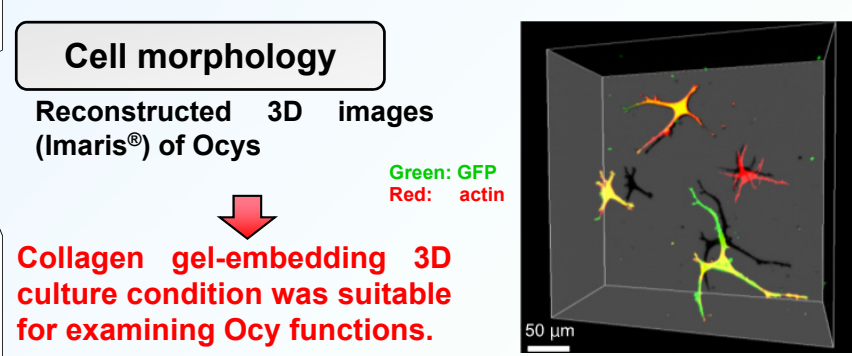

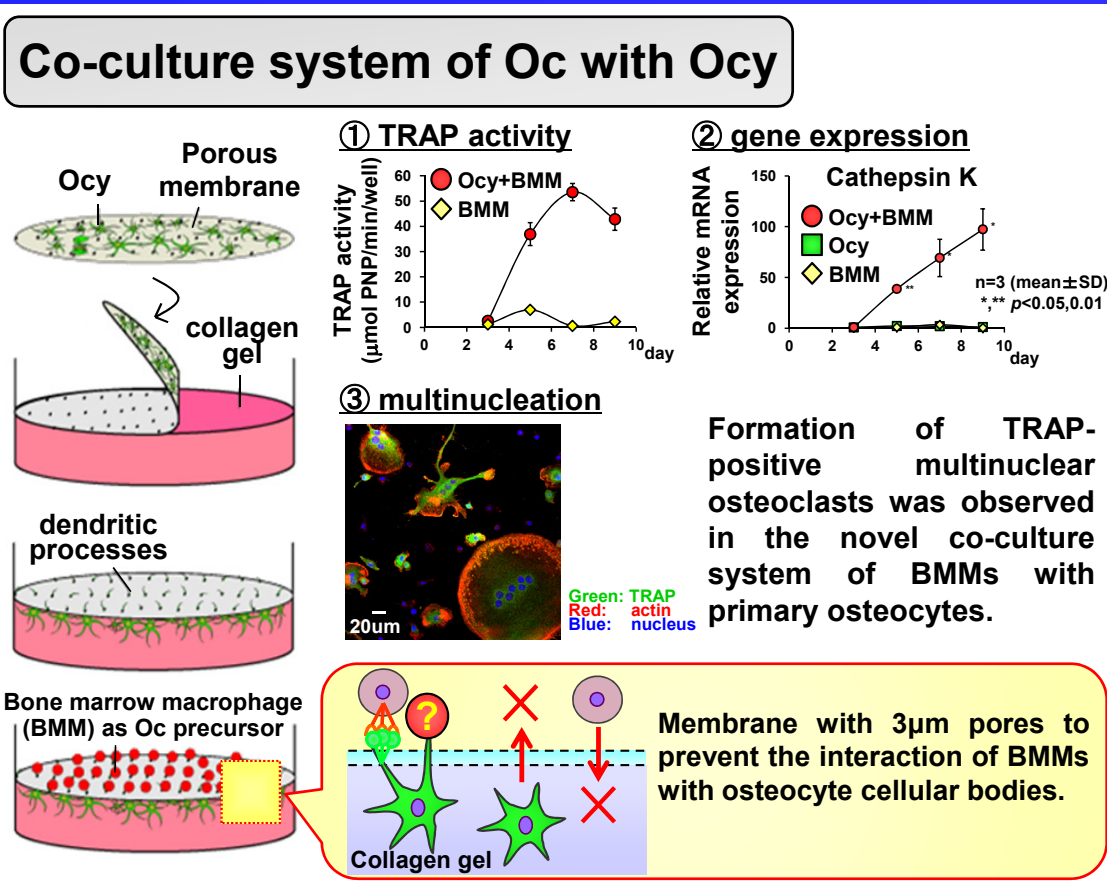

2. Interaction between Osteocyte and Osteoclast

Soluble form?

(1) Suppression of soluble RANKL production using TIMP-2

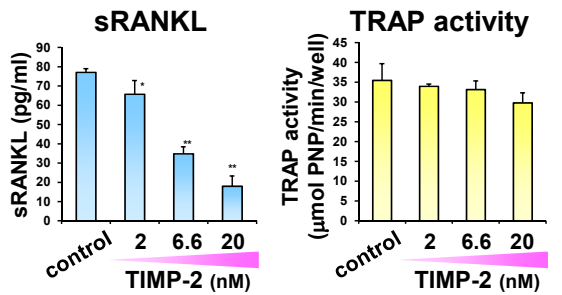

(2) Addition of soluble RANKL

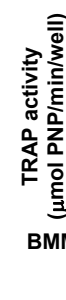

\section{(1)}
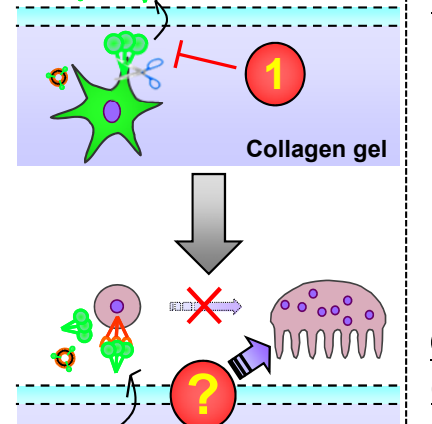
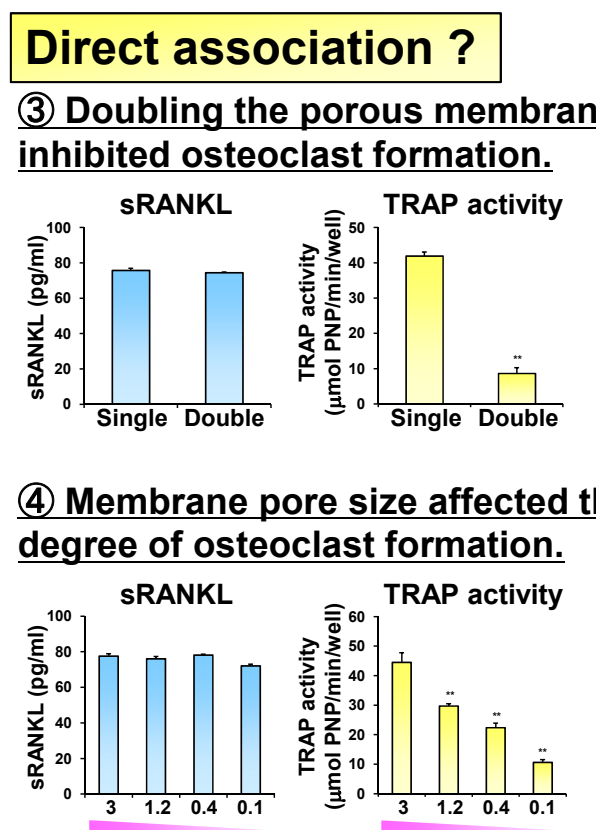

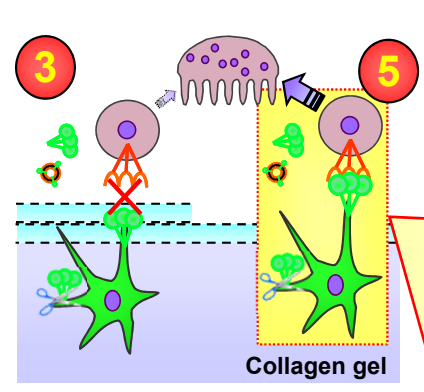

(5) Dendritic processes of Ocy directly contacted with BMMs

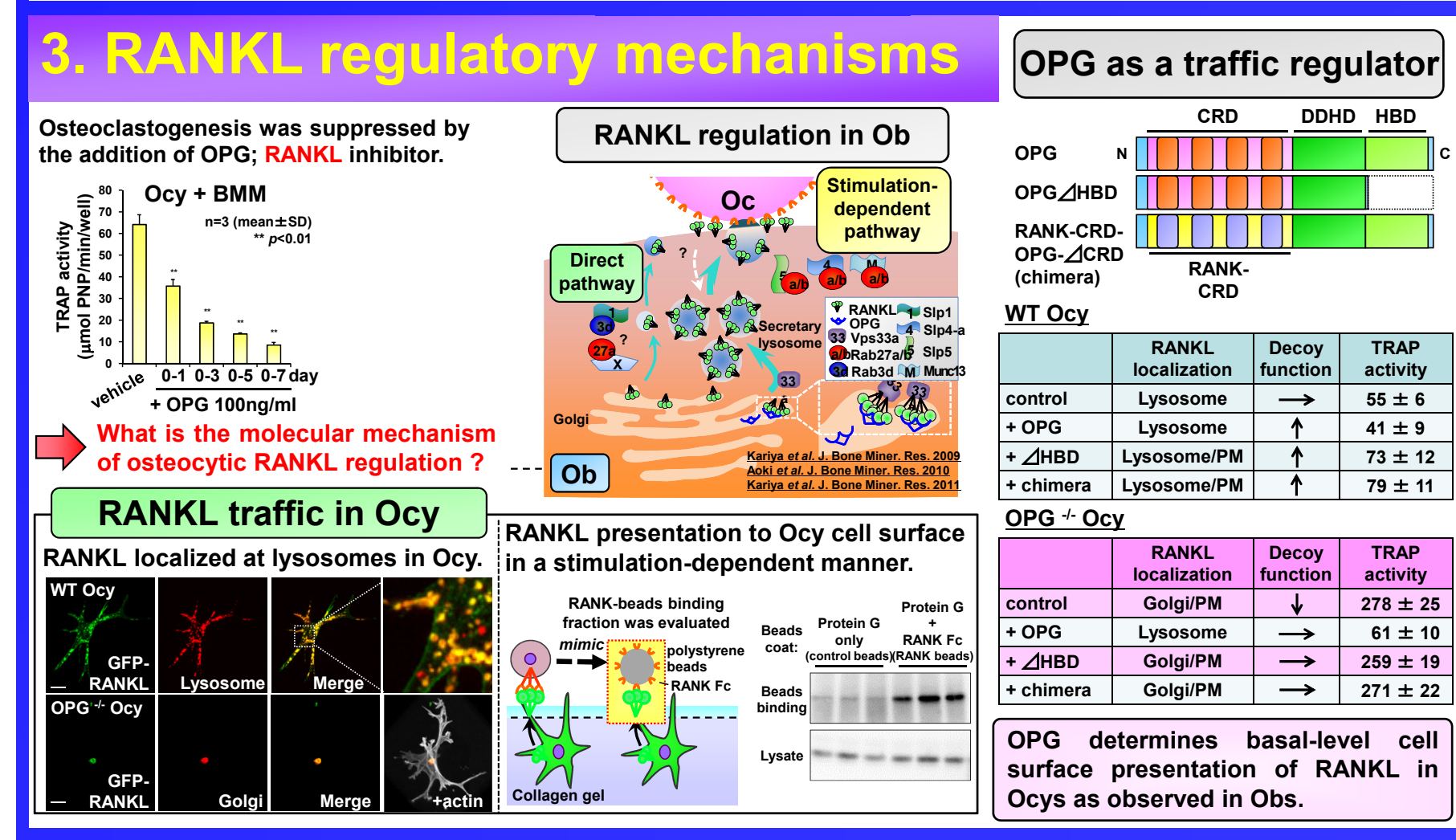

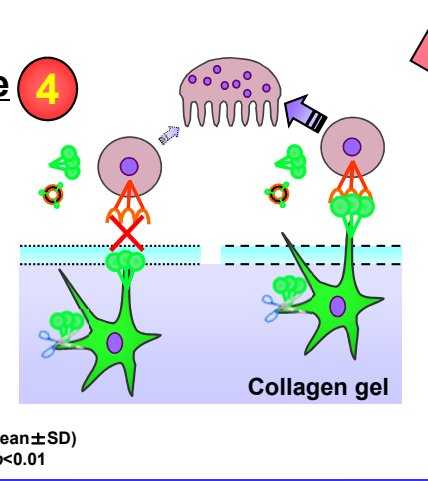

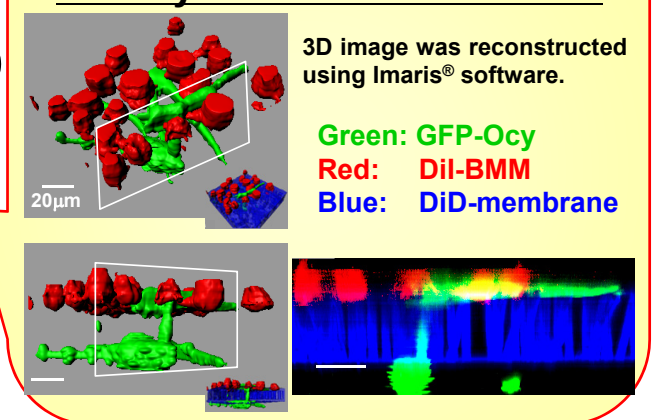

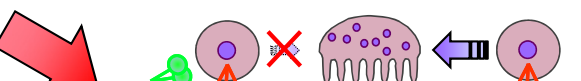

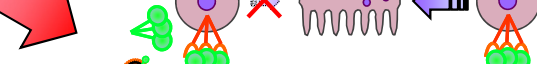

o
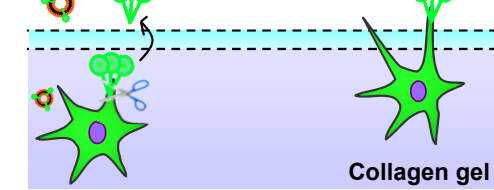

Osteoclastogenesis supported by Ocy may be largely dependent on direct cell-to-cell contact at the extremities of dendritic processes.

\section{Summary}

1. The novel co-culture system of Ocys with BMMs using collagen matrix and porous membrane was established.

2. Osteoclastogenesis may be largely supported by direct cell-to-cell contact between Ocys and BMMs at the Ocy dendritic processes.

3. OPG functions as a traffic regulator of RANKL in Ocys as well as in Obs.

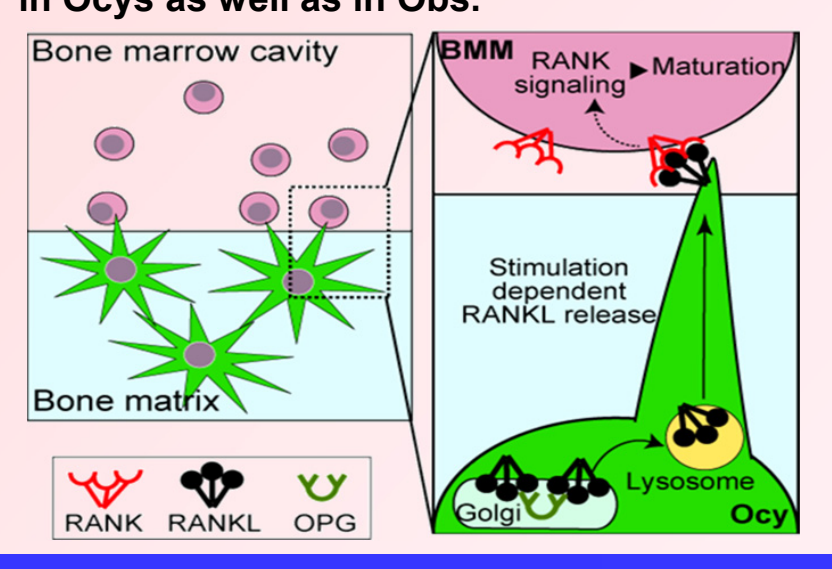

